Brain Research. Molecular Brain Research (1999) 70: 231-241

\title{
L-Arginine Uptake, the Citrulline-NO Cycle and Arginase II in the Rat Brain: An in Situ Hybridization Study.
}

\author{
Olivier Braissant ${ }^{1}$, Tomomi Gotoh ${ }^{2}$, Marc Loup ${ }^{1}$, Masataka Mori ${ }^{2}$ \\ and Claude Bachmann ${ }^{1}$.
}

1 Central Clinical Chemistry Laboratory, University Hospital, CH-1011 Lausanne, Switzerland.

${ }^{2}$ Department of Molecular Genetics, Kumamoto University School of Medicine, Kumamoto 860-0811, Japan.

Correspondence to :

Olivier Braissant

Central Clinical Chemistry Laboratory,

University Hospital,

CH-1011 Lausanne, Switzerland

Tél : (+41.21) 314.41.52

Fax : (+41.21) 314.42.88

e-mail : Olivier.Braissant@chuv.hospvd.ch

24 pages, including 2 tables and 4 figures.

\section{Abstract}


Nitric oxide (NO) is synthesized from a unique precursor, arginine, by nitric oxide synthase (NOS). In brain cells, arginine is supplied by protein breakdown or extracted from the blood through cationic amino acid transporters (CATs). Arginine can also be recycled from the citrulline produced by NOS activity, through argininosuccinate synthetase (AS) and argininosuccinate lyase (AL) activities, and metabolized by arginase. NOS, AS and AL constitute the so-called citrulline-NO cycle. In order to better understand arginine transport, recycling and degradation, we studied the regional distribution of cells expressing CAT1, CAT3, AS, AL, neuronal NOS (nNOS) and arginase II (AII) in the adult rat brain by nonradioisotopic in situ hybridization. CAT1, AL and AII presented an ubiquitous neuronal and glial expression, whereas CAT3 and AS were confined to neurons. nNOS was restricted to scattered neurons and a few brain nuclei and layers. We demonstrate by this study that cells expressing nNOS all appear to express the entire citrulline-NO cycle, whereas numerous cells expressing AL do not express AS. The differential expression of these genes within the same anatomical structure could indicate that intercellular exchanges of citrulline-NO cycle metabolites are relevant. Thus vicinal interactions should be taken into account to study their regulatory mechanisms.

Keywords: brain, arginine, nitric oxide, system $\mathrm{y}^{+}$, arginase, argininosuccinate, in situ hybridization.

Theme B: Cellular and Molecular Biology.

Topic: Gene structure and function: general.

\section{Introduction}


Nitric oxide (NO) is an important cell-cell signalling molecule in the central nervous system (CNS) [14]. It is synthesized from a unique precursor, L-arginine, through nitric oxide synthase (NOS) activity [30]. The NO production capacity is dependent on the intracellular arginine concentration. Arginine is also utilized for the synthesis of proteins and creatine, and is metabolized by arginase to ornithine and urea (Figure 1). There is no de novo synthesis of arginine in CNS, since the mitochondrial urea cycle enzymes carbamoylphosphate synthetase and ornithine transcarbamylase are not expressed. For their needs in arginine, brain cells depend on protein breakdown and on specific transporters to import arginine from the blood or transfer it from cell to cell [26]. Arginine can also by recycled from the citrulline generated by the NOS activity, in a reaction catalized by the cytosolic argininosuccinate synthetase (AS) and argininosuccinate lyase (AL). This constitutes the so-called citrulline-NO cycle or arginine-citrulline cycle (Figure 1, reviewed in [42]). In the brain, arginine levels and NO production appear to be at a pivotal place in the pathophysiology of hyperammonemic disorders [32,33], where, in astrocytes of aggregating neural cell cultures, the expression of AS and AL genes is induced [7].

L-arginine is transported through cell membrane by carriers designated as system $\mathrm{y}^{+}$[26]. In CNS and neural cell cultures, the system $\mathrm{y}^{+}$was identified at the blood brain barrier [40], in primary cultured astrocytes [35], in cultured neurons from neocortex and cerebellum [46] as well as in synaptosomes extracted from cortex and cerebellum [1]. System $\mathrm{y}^{+}$carriers are a family of cationic amino acid transporters called CATs [26]. Up to now, four different CAT proteins have been identified. CAT1 is considered to be ubiquitously expressed except in liver [22], CAT2(B) is expressed in different tissues including brain but absent in liver [25], CAT2A, an alternatively spliced variant of CAT2(B), is restricted to liver [11], and CAT3 was recently isolated from the rodent brain $[17,18]$. CAT 1, 2(B) and 3 present a high affinity for arginine (apparent $\mathrm{Km}$ around $100 \mu \mathrm{M}$ ), whereas CAT2A has a much lower affinity profile (apparent $\mathrm{Km}$ of 2 to $5 \mathrm{mM}$ ). CAT1 and CAT2(B) have been described in whole brain homogenates $[22,25]$. However, data on the precise identification and localization of CAT transporters responsible for the arginine trafficking between the different cell types of the brain are still lacking, except for the neurospecific CAT3 which seems to be expressed in restricted areas of the brain $[17,18]$. 
In the brain, the recycling of arginine from citrulline has been proposed to occur in neurons [10]. AS and AL activities have been described since a long time in CNS [34]. In the rat brain, AS has been localized by immunohistochemistry in specific subsets of neurons, whereas AL was found in a specific glial subtype of cerebellum as well as neurons of the whole brain $[4,27,28]$. Thus, AS and AL appear to be expressed predominantly in neurons in the normal brain. However, AS and AL genes were found induced by cyclic AMP and glucocorticoids in primary astrocyte cultures [21] and by hyperammonemia in aggregating brain cell culture astrocytes [7]. The AS protein was recently found increased in cultured primary astrocytes after treatment with lipopolysaccharide (LPS) and interferon- $\gamma$ (IFN $\gamma$ ) [36].

Three NOS isoforms have been described, namely neuronal NOS (nNOS), endothelial NOS (eNOS) and inducible NOS (iNOS) (reviewed in [23]). nNOS is constitutively expressed in CNS, and recent studies described the precise distribution of nNOS mRNA and protein in discrete populations of neurons of the adult rat brain [8,19]. eNOS plays also a role in CNS, as mice doubly disrupted for nNOS and eNOS present a reduction of long term potentiation in the CA1 region, whereas single nNOS disrupted mice do not [37]. eNOS seems restricted to the brain vasculature [38]. There is increasing evidence of the involvement of iNOS in aging CNS and neurodegenerative processes [43,45].

Despite the diffuse expression of the liver type arginase I (AI) in CNS [28], arginase activity in the brain seems predominantly due to the mitochondrial arginase II (AII) [16,44]. It was proposed that AII acts in combination with arginine biosynthetic pathways in order to modulate arginine levels [44], and that it is involved in down-regulation of NO synthesis [15]. Nothing is known on the precise cellular localization of AII in CNS.

For better understanding the CNS regulation of arginine transport, recycling and degradation, a detailed localization study of the brain expression of these genes was needed first. In this study, we present the precise distribution of CAT1, CAT3, AS, AL, nNOS and AII mRNAs in the brain of adult rat by non-radioisotopic in situ hybridization (ISH).

\section{Materials and Methods}




\section{cDNAs, plasmids and riboprobes synthesis.}

Specific cDNA fragments for rat AS (AS [41], nt 340 to 790), AL (AL [2], nt 880 to 1350), CAT1 (CAT1 [40], nt 202-804), CAT3 (CAT3 [17], nt 795-1839), nNOS (nNOS [9], nt 3002150) and arginase II (AII [20], nt 86-1211) were obtained by reverse transcription coupled to PCR, and cloned in the vector pGEM-3Zf(+) into the HincII site. The resulting plasmids were linearized as follows: pGEM-AS, pGEM-AL, pGEM-CAT1, pGEM-CAT3, pGEM-nNOS and pGEM-AII with HindIII to yield antisense probes for CAT1 and CAT3, and sense probes for AS, AL, nNOS and AII; pGEM-AS, pGEM-AL, pGEM-CAT1, pGEM-CAT3, and pGEM-nNOS with XbaI to yield antisense probes for AS, AL and nNOS, and sense probes for CAT1 and CAT3; pGEM-AII with EcoRI to yield antisense probe for AII. The linearized plasmids were gel-purified and used as templates for digoxigenin labelled riboprobe synthesis, using T7- or SP6-RNA polymerases (Promega, Madison, WI). The transcription mixture included 1mM ATP, GTP and CTP, 0.7mM UTP and 0.3mM digoxigenin-UTP (Roche Molecular Biochemicals, Basel, Switzerland). After digestion of the DNA templates by RQI-DNase (Promega, Madison, WI), the RNA probes were purified by two ethanol precipitations and resuspended in diethylpyrocarbonate (DEPC, Fluka, Buchs, Switzerland) treated water.

\section{In situ hybridization and immunohistochemistry}

3 male adult Sprague-Dawley rats (300 g, BRL, Basel, Switzerland) were deeply anesthetized (10 mg/rat Ketalar, Parke davis, USA), rapidly perfused with DEPC-treated PBS and sacrified by decapitation. Their brain was extracted within 2 minutes, immediately embedded in tissue freezing medium (Jung, Nussloch, Germany) and frozen in isopentane and dry ice. Brains were kept at $-80^{\circ} \mathrm{C}$ until used, then cut and analyzed by ISH as described [6]. Briefly, cryosections $12 \mu \mathrm{m}$ thick were postfixed 10 minutes in 4\% paraformaldehyde-PBS, washed 2x15 minutes in PBS containing 0.1\% fresh DEPC and equilibrated 15 minutes in 5xSSC. Sections were then hybridized $\left(58^{\circ} \mathrm{C}\right.$ for $40 \mathrm{~h}$ in $5 \mathrm{xSSC}, 50 \%$ formamide and $40 \mu \mathrm{g} / \mathrm{ml}$ salmon sperm DNA) with digoxigenin-labelled antisense and sense riboprobes (400 ng/ml) for rat AS, AL, CAT1, CAT3, nNOS and AII. Sections were washed (30 minutes in 2xSSC at room temperature, 1 hour in $2 \mathrm{xSSC}$ at $65^{\circ} \mathrm{C}$, 1 hour in $0.1 \mathrm{xSSC}$ at $65^{\circ} \mathrm{C}$ ) and stained with alkaline-phosphatase (15 hours at room temperature) as described [6]. After staining, sections were dehydrated and mounted (Eukitt, O.Kindler Gmbh \& Co., Freiburg, Germany). The 
specificity of hybridization was ascertained by the use of sense probes for AS, AL, CAT1, CAT3, nNOS and AII genes having the same length, GC content and activity of digoxigenin labelling as the corresponding antisense probes. In each ISH experiment, a section hybridized with a specific antisense probe was always preceded or followed by an adjacent section hybridized with the corresponding sense control probe.

Immunohistochemical labelling of neurons, astrocytes and oligodendrocytes was carried out using monoclonal antibodies directed against Microtubule Associated Protein 2 (MAP2), Glial Fibrillary Acidic Protein (GFAP) (Roche Molecular Biochemicals, Basel, Switzerland) and Myelin Basic Protein (MBP) (Boehringer Ingelheim, Germany), respectively. MAP2, GFAP and MBP immunohistochemical stainings were alternated with the ISH stainings, generally in the following order: ISH for CAT1 (antisense and sense) / MAP2 / ISH for CAT3 and AS (antisense and sense) / GFAP / ISH for AL and nNOS (antisense and sense) / MBP / ISH for AII (antisense and sense). When needed, the order of immunohistochemical stainings was modified to precise the cell identification of a transcript localized by ISH. $12 \mu \mathrm{m}$ thick cryosections were fixed 1 hour in 4\% paraformaldehyde-PBS at room temperature, then washed 3x5 minutes in PBS. Primary antibody (diluted 1:100 in PBS containing 1\% bovine serum albumin and $0.3 \%$ Triton X100) was applied overnight at $4^{\circ} \mathrm{C}$. Sections were rinsed $3 x 5$ minutes in PBS, and an anti-mouse IgG (Sigma) bridging antibody (diluted 1:100 in PBS containing $1 \%$ bovine serum albumin and $0.3 \%$ Triton X100) was applied for 1 hour at room temperature. Sections were rinsed $3 \times 5$ minutes in PBS, and the complex alkaline phosphatase / anti-alkaline phosphatase (Sigma, diluted 1:100 in PBS containing 1\% bovine serum albumin and $0.3 \%$ Triton X100) was applied for 1 hour at room temperature. Sections were rinsed 3x5 minutes in PBS, and stained and mounted as described above for ISH.

\section{Histological analysis}

Sections were observed and photographed on an Olympus microscope (Olympus Opticals, Japan). No divergence of results was observed between the three male rats analysed. Brain structures were identified according to Paxinos and Watson [31]. 


\section{Results}

A semi-quantitative description of CAT1, CAT3, AS, AL, nNOS and AII mRNA levels in the different brain structures in given in Table 1. The specificity of hybridization was ascertained by the absence of signal obtained with sense probes for CAT1, CAT3, AS, AL, nNOS and AII genes (Fig. 2-e,g,i,k,m,o).

\section{Cationic amino acid transporter 1 (CAT1)}

The CAT1 mRNA was found in both neurons and glial cells of the rat brain, and its cellular localization was perinuclear. In telencephalon, CAT1 was expressed throughout the neocortex (Fig. 2-b,d), in the putamen, globus pallidum, nucleus accumbens, pyriform cortex and its transition towards the amygdala nuclei (Fig. 2-k). It was further detected in the anterior olfactory nucleus and the anterior intrabulbar commissure. In hippocampus, the CAT1 transcript was localized at high levels in the CA 1 to 3 layers, the dentate gyrus as well as the polymorphic and molecular layers, where it stained both neurons and astrocytes (Fig. 3a,c,d,e). Most structures of diencephalon, midbrain, pons and medulla showed the CAT1 transcript with particularly high levels in the gigantocellular reticular nucleus (Fig. 4a). In cerebellar cortex, CAT1 was expressed in the granular and molecular layers as well as in the Purkinje cells (Fig. 4-c). CAT1 was also found in the deep nuclei of cerebellum. The CAT1 mRNA was expressed at low levels in white matter structures of corpus callosum and cerebellum, in cells identified as oligodendrocytes by MPB staining (Fig. 2-b, 4-c and data not shown). CAT1 transcript was also revealed in the choroid plexus (data not shown).

\section{Cationic amino acid transporter 3 (CAT3)}

The CAT3 mRNA was detectable in neurons only, and was particularly high in ventral basal ganglia, ventral hypothalamus and midbrain nuclei. In every case, the CAT3 transcript localization was diffuse and included neuronal prolongations. In telencephalon, the CAT3 mRNA was found at low levels in neocortex, with higher levels in layers II and III (Fig. 2-f). The signal was diffuse and included fibers, with some rare neurons expressing it at higher levels (Fig. 2-f). High expression was also observed in the entorhinal cortex and subiculum (data not shown). The external capsule of the putamen presented a high level of CAT3 transcript, whereas low levels were found in its central part. High levels of CAT3 mRNA were found in ventral striatum and islands of Calleja (Fig. 2-l). CAT3 gene expression was 
also found in the anterior olfactory nucleus, but not in the anterior commissure. In hippocampus, CAT3 transcript was localized at high levels in CA1, but less in CA3. The dentate gyrus presented a high level of expression, and rare neurons of the polymorphic and molecular layers were positive (Fig. 3-f). In diencephalon, the CAT3 mRNA was found at low levels in thalamic and hypothalamic nuclei (Table 1). The hypothalamic premammillary ventral nucleus showed scattered neurons with high levels of CAT3 transcript. In midbrain, CAT3 was expressed in the superior colliculus, but barely detectable in the inferior colliculus. Higher levels were found in red nucleus and substantia nigra. In pons and medulla, a diffuse and low signal was observed, with a high level of expression in vestibular and reticulate formation nuclei. In cerebellar cortex, CAT3 mRNA was highly expressed in the granular layer and the Purkinje cells, and at low levels in the molecular layer (Fig. 4-d). It was expressed at very low levels in the deep nuclei of cerebellum. The CAT3 mRNA was absent from any white matter structure (Fig. 4-d). CAT3 transcript was expressed in the choroid plexus (data not shown).

\section{Argininosuccinate synthetase (AS)}

In the whole rat brain, the AS mRNA was localized in neurons and not detectable in glial cells. In telencephalon, the AS mRNA was found in most layers of neocortex, including higher levels in scattered neurons as well as in the deepest cells of layer VI (Fig. 2-c,h). Scattered neurons with high levels of AS mRNA were localized in putamen, septum, globus pallidum, nucleus accumbens, islands of Calleja, pyriform cortex and amygdala. AS was also expressed in anterior olfactory nuclei. In hippocampus, the AS transcript was localized at low levels in the dentate gyrus, and barely detectable in CA, polymorphic and molecular layers (Fig. 3-g). Most nuclei of diencephalon expressed the AS mRNA. In midbrain, it was expressed at very low levels, together with scattered neurons showing higher expression. The AS mRNA was expressed in most structures of pons and medulla, with particularly high levels in the gigantocellular reticular nucleus (Fig. 4-b). In cerebellar cortex, the AS transcript was highly expressed in the granular and molecular layers, but was absent from the Purkinje cells (Fig.4-f). It could not be detected in the deep nuclei of cerebellum nor in white matter (Fig. 2-c and 4f). It was also absent from the choroid plexus (data not shown).

\section{Argininosuccinate lyase (AL)}


The AL mRNA was found at low level of expression in most structures of the rat brain, both in neurons and astrocytes, but seemed absent from oligodendrocytes. In most of the brain cells, its localization was perinuclear. However, some brain areas presented also a cell process localization of the AL transcript. This was particularly the case in neocortex (diffuse signal, Fig. 2-j). AL mRNA was also observed in ventral striatum, islands of Calleja and nucleus accumbens (Fig. 2-m), as well as in the anterior olfactory nucleus. In hippocampus, the AL transcript was localized at low levels in the CA layer and the dentate gyrus, and barely detectable in the polymorphic and molecular layers (Fig. 3-h). The AL mRNA was present at low levels in diencephalon, midbrain, pons and medulla, with peculiar expression in vestibular and solitary tract nuclei. In cerebellar cortex, it was expressed at low levels in the granular and molecular layers, as well as the Purkinje cells (Fig. 4-e). No expression could be observed in the deep nuclei of cerebellum. Except for a few astrocytes in corpus callosum, the AL mRNA was absent from any white matter structure (Fig. 4-e). The AL transcript was expressed in the choroid plexus (data not shown).

\section{Neuronal Nitric Oxide Synthase (nNOS)}

In most structures of the brain, the nNOS mRNA was not detectable or expressed at very low levels, except in scattered neurons showing very high levels of transcript localized also in the cell processes (Fig. 2-l). Glial cells did not express nNOS transcript. In telencephalon, scattered neurons with very high levels of nNOS transcript were localized in neocortex (Fig. 2-1), putamen, globus pallidum, nucleus accumbens and pyriform cortex. The islands of Calleja and the anterior olfactory nuclei were strongly positive for nNOS mRNA. In hippocampus, nNOS transcript was very low in the dentate gyrus and the CA layer, and high in scattered neurons throughout the polymorphic and molecular layers (Fig. 3-i). In diencephalon, it was absent from thalamus, except from the stria medullaris thalami. In hypothalamus, ventromedial hypothalamic and premammillary nuclei were strongly positive. In midbrain, the nNOS transcript was predominantly found in the superficial gray layer of the superior colliculus and in the dorsal cortex of the inferior colliculus. It was also found in the red nucleus. In pons and medulla, nNOS mRNA was localized in pontine nucleus, giant neurons of the solitary tract, as well as in the subpedunculate tegmentum and subcoeruleus nuclei. In cerebellum, it was highly expressed in the granular and molecular layers, but was absent from the Purkinje cells (Fig. 4-g). It was expressed at low levels in scattered neurons of the deep nuclei of cerebellum. nNOS transcript was absent from choroid plexus (data not 
shown).

\section{Arginase II (AII)}

The AII mRNA was found in most structures of the rat brain, both in neurons and glial cells (Fig. 2-n and 2-j). Its cellular localization was perinuclear. In telencephalon, the arginase II gene was expressed throughout neocortex (Fig. 2-n) and corpus callosum, as well as putamen. High levels were observed in ventral striatum, nucleus accumbens and islands of Calleja (Fig. 2-n), pyriform cortex and its transition towards the amygdala nuclei. In hippocampus, the arginase II transcript was more expressed in CA1 than CA3 (Fig. 3-b). The dentate gyrus as well as neurons and astrocytes of the polymorphic and molecular layers expressed high levels of AII mRNA (Fig. 3-b,c,d,j). The AII transcript was localized at low levels in most structures of diencephalon, midbrain, pons and medulla. In cerebellar cortex, it was highly expressed in the granular and molecular layers, but was absent from the Purkinje cells (Fig. 4-h). It was also localized in numerous cell bodies of the cerebellar white matter, in cells identified as oligodendrocytes by MBP staining (Fig. 4-h and data not shown). The AII gene was expressed at low levels in the deep nuclei of cerebellum. AII transcript was also expressed in the choroid plexus (data not shown). 


\section{Discussion}

\section{Expression of CAT1, CAT3, AS, AL, nNOS and AII}

Previous reports have described the presence of CAT1 in the rodent brain by northern blotting and RT-PCR experiments [18,22,25], but a precise distribution of CAT1 expression in CNS is lacking. We show in this study that the CAT1 mRNA is found both in neurons and astrocytes of the whole brain, and most probably in oligodendrocytes as well. CAT3 has been described in the rodent brain, and its mRNA was mostly localized in diencephalon and midbrain $[17,18]$, which fits with the results we obtained in this study. In addition, we show that the CAT3 mRNA is predominantly expressed by neurons, and that CAT3 mRNA is also expressed in telencephalon, brainstem and cerebellum. The presence of AS and lyase AL enzyme activities and gene expression are known since a long time in CNS [4,27,28,34]. Our findings by ISH demonstrate the expression of AS mRNA in neurons, but not in glial cells, and a diffuse and low signal for AL transcript both in neurons and astrocytes. The distribution of AS and AL transcripts we found fits well with previous immunohistochemical studies $[4,27,28]$. We have further demonstrated the expression of AL mRNA in the Purkinje cells of cerebellar cortex, which validates data at the protein levels where the signal specificity obtained has been described as dubious [28]. Our ISH study has shown that in most part of the rat brain, nNOS mRNA is expressed at high levels in scattered neurons of the whole brain, in the cortex of cerebellum (molecular and granular layers), as well as in few nuclei of diencephalon, midbrain, brainstem. Isolated neurons with lower levels of expression were found also in diverse part of CNS. Our data are in accordance with previous reports on the adult rodent brain, either at the transcript or the protein level $[8,19]$. Data about arginase expression in CNS are contradictory. Whereas AI, the liver form, was described by immunohistochemistry and localized diffusely in CNS [28], other studies could not detect the AI mRNA in different brain regions [16], but could detect the non-hepatic AII transcript $[16,44]$. To our knowledge, no data about the AII localization in the brain have been published. Our ISH study shows that the AII mRNA is distributed uniformly in CNS and found in most neurons and astrocytes, except for a few cells like the Purkinje neurons of the cerebellar cortex which do not express AII mRNA.

\section{Localization of transcripts in the CNS cells}

All transcripts we have studied were localized or concentrated in the perinuclear region of brain cells. However, an interesting finding is the presence of CAT3, AL and nNOS 
transcripts in the cell processes. This is easily detectable for nNOS, due to the isolation of the neurons expressing it, and more diffuse for CAT3 and AL, expressed by much more numerous cells, which however present a diffuse mRNA localization in dense fiber regions, particularly in the cortex of telencephalon. A discrimination between axons and dendrites (CAT3, AL) or astrocyte processes (AL) is however difficult. For nNOS, the expression of the mRNA in the processes appears to be dendritic. Thus, CAT3, AL and nNOS mRNAs might be transported along the cell processes, in order to allow the brain cells to translate these proteins at the site they are needed, in a similar fashion as described in dendrites for the MAP2 mRNA [13]. This would allow the cell to respond rapidly to immediate peripheral needs in these proteins. It does not exclude an anterograde transport of newly translated CAT1, CAT3, AS, AL, nNOS and AII proteins towards cell processes, as observed for example for AS in previous immunohistochemical studies [4,27].

\section{Patterns of cellular expression, arginine uptake and metabolism in brain cells}

The presence of $\mathrm{y}^{+}$carrier system has been shown at the blood brain barrier [40], in cultured neurons [46], in synaptosomes [1] and in primary astrocyte cultures [35]. The expression of CAT1 at the blood brain barrier and choroid plexus [40] as well as our findings showing its expression in the whole brain (Table 2) renders CAT1 the prime candidate for basic arginine trafficking between brain cells. CAT3 on the other hand seems restricted to neurons, and could more specifically be responsible for arginine uptake and exchange by neurons. In CNS, free L-arginine seems predominantly localized in glial cells [3], which can release arginine upon excitatory amino acid receptor activation [12]. This suggests that arginine trafficking between blood and astrocytes, between astrocytes and neurons, as well as between neurons themselves, is important not only for protein synthesis, but also in the cellular response to stimulation. Another form of CAT, CAT2(B), could be responsible of arginine trafficking in glial cells; it was localized in brain by northern blotting $[18,25]$ and found up-regulated in primary astrocytes upon LPS and IFN $\gamma$ treatment [39]. Further work should localize precisely the CAT2(B) expression in the brain.

As shown in Table 1, patterns of expression of the genes we studied differ, depending on cell types and localization. However, even within the same anatomical region and morphological cell types, differences of expression were found. The data are summarized in Table 2. Coexpression of all genes of the citrulline-NO cycle is a prerequisite for assuming that cells do not depend for their NO production on intercellular arginine transport. Such a pattern can 
only be found in scattered neurons mainly localized in telencephalon, midbrain and brainstem, in a few nuclei of hypothalamus and brainstem, as well as in the cerebellar cortex. Neurons expressing AS and AL but not nNOS might be involved in the recycling of the citrulline produced by NO synthesizing cells into arginine. This raises the question of citrulline trafficking between brain cells and uptake of arginine by NOS expressing cells. The expression of eNOS and iNOS in CNS as well as the induction of iNOS in pathological circumstances suggest that the citrulline-NO cycle might be essential also in cells not expressing nNOS. Neurons and glial cells expressing AL but lacking AS mRNAs raise the question of argininosuccinate release, trafficking and potential role as messenger or neurotransmitter in or between brain cells, as already suggested by similar observations [29]. Alternatively, the dormant AS gene could be induced in these cells under specific pathological conditions, as it is up-regulated in primary astrocytes upon LPS and IFN $\gamma$ treatment [36] and, together with the AL gene, in astrocytes of reaggregated brain cell cultures exposed to ammonium chloride [7].

Our findings indicate that most cells of the brain appear able to regulate their arginine levels by AII. Purkinje neurons are an exception to this. AII is proposed to act in combination with arginine biosynthetic pathways in order to modulate arginine levels [44], to decrease NO synthesis or to block NOS dependent apoptosis, as suggested by recent works on macrophage cell lines [15]. However, the absence of NOS expression in most brain cells points to the fact that arginine is used for more general purposes, such as protein and creatine synthesis. In CNS, astrocytes are the main cells synthesizing polyamines [24], which have ornithine as substrate. Astrocytes have been shown as the predominant locus of expression of UT3, a urea transporter thought to participate in the elimination of urea by the brain [5]. These findings could be put in relation with the astrocytic expression of AII, which metabolizes arginine to ornithine and urea.

Our data suggest that all intermediates of the citrulline-NO cycle might be exchanged between brain cells in order to ensure their needs in NO and arginine. The precise description of the restricted expression of CAT 1 and 3, AS, AL, nNOS and AII by specific cells is a prerequisite for understanding the citrulline-NO cycle and the roles of arginine in the brain, which will have to take into account interactions between cells in close vicinity. 


\section{References}

1. Aldridge, C.R. and Collard, K.J., The characteristics of arginine transport by rat cerebellar and cortical synaptosomes, Neurochem.Res., 21 (1996) 1539-1546.

2. Amaya, Y., Matsubasa, T., Takiguchi, M., Kobayashi, K., Saheki, T., Kawamoto, S. and Mori, M., Amino acid sequence of rat argininosuccinate lyase deduced from cDNA, J.Biochem., 103 (1988) 177-181.

3. Aoki, E., Semba, R., Mikoshiba, K. and Kashiwamata, S., Predominant localization in glial cells of free L-arginine. Immunocytochemical evidence, Brain Res., 547 (1991) 190-192.

4. Arnt-Ramos, L.R., O'Brien, W.E. and Vincent, S.R., Immunohistochemical localization of argininosuccinate synthetase in the rat brain in relation to nitric oxide synthase-containing neurons, Neuroscience, 51 (1992) 773-789.

5. Berger, U.V., Tsukaguchi, H. and Hediger, M.A., Distribution of mRNA for the facilitated urea transporter UT3 in the rat nervous system, Anatomy \& Embryology, 197 (1998) 405414.

6. Braissant, O., Foufelle, F., Scotto, C., Dauça, M. and Wahli, W., Differential expression of peroxisome proliferator-activated receptors (PPARs): tissue distribution of PPAR-alpha, beta, and -gamma in the adult rat, Endocrinology, 137 (1996) 354-366.

7. Braissant, O., Honegger, P., Loup, M., Iwase, K., Takiguchi, M. and Bachmann, C., Hyperammonemia: Regulation of Argininosuccinate Synthetase and Argininosuccinate Lyase Genes in Aggregating Cell Cultures of Fetal Rat Brain, Neuroscience Letters, (1999) in press.

8. Bredt, D.S., Glatt, C.E., Hwang, P.M., Fotuhi, M., Dawson, T.M. and Snyder, S.H., Nitric oxide synthase protein and mRNA are discretely localized in neuronal populations of the mammalian CNS together with NADPH diaphorase, Neuron, 7 (1991) 615-624.

9. Bredt, D.S., Hwang, P.M., Glatt, C.E., Lowenstein, C., Reed, R.R. and Snyder, S.H., Cloned and expressed nitric oxide synthase structurally resembles cytochrome P-450 reductase, Nature, 351 (1991) 714-718.

10. Chen, F.Y. and Lee, T.J., Arginine synthesis from citrulline in perivascular nerves of cerebral artery, J.Pharmacol.Exp.Therapeut., 273 (1995) 895-901.

11. Closs, E.I., Albritton, L.M., Kim, J.W. and Cunningham, J.M., Identification of a low affinity, high capacity transporter of cationic amino acids in mouse liver, J.Biol.Chem., 268 (1993) 7538-7544.

12. Do, K.Q., Benz, B., Grima, G., Gutteck-Amsler, U., Kluge, I. and Salt, T.E., Nitric oxide precursor arginine and S-nitrosoglutathione in synaptic and glial function, Neurochem.Int., 29 (1996) 213-224.

13. Garner, C.C., Tucker, R.P. and Matus, A., Selective localization of messenger RNA for cytoskeletal protein MAP2 in dendrites, Nature, 336 (1988) 674-677. 
14. Garthwaite, J. and Boulton, C.L., Nitric oxide signaling in the central nervous system, Ann.Rev.Physiol., 57 (1995) 683-706.

15. Gotoh T. and Mori M., Arginase II downregulates nitric oxide (NO) production and prevents NO-mediated apoptosis in murine macrophage-derived RAW 264.7 cells, J.Cell Biol., 144 (1999) 427-434.

16. Gotoh, T., Araki, M. and Mori, M., Chromosomal localization of the human arginase II gene and tissue distribution of its mRNA, Biochem.Biophys.Res.Commun., 233 (1997) 487491.

17. Hosokawa, H., Sawamura, T., Kobayashi, S., Ninomiya, H., Miwa, S. and Masaki, T., Cloning and characterization of a brain-specific cationic amino acid transporter, J.Biol.Chem., 272 (1997) 8717-8722.

18. Ito, K. and Groudine, M., A new member of the cationic amino acid transporter family is preferentially expressed in adult mouse brain, J.Biol.Chem., 272 (1997) 26780-26786.

19. Iwase, K., Iyama, K., Akagi, K., Yano, S., Fukunaga, K., Miyamoto, E., Mori, M. and Takiguchi, M., Precise distribution of neuronal nitric oxide synthase mRNA in the rat brain revealed by non-radioisotopic in situ hybridization, Mol.Brain Res., 53 (1998) 1-12.

20. Iyer, R.K., Bando, J.M., Jenkinson, C.P., Vockley, J.G., Kim, P.S., Kern, R.M., Cederbaum, S.D. and Grody, W.W., Cloning and characterization of the mouse and rat type II arginase genes, Molecular Genetics \& Metabolism, 63 (1998) 168-175.

21. Jackson, M.J., Zielke, H.R. and Zielke, C.L., Induction of astrocyte argininosuccinate synthetase and argininosuccinate lyase by dibutyryl cyclic AMP and dexamethasone, Neurochem.Res., 21 (1996) 1161-1165.

22. Kim, J.W., Closs, E.I., Albritton, L.M. and Cunningham, J.M., Transport of cationic amino acids by the mouse ecotropic retrovirus receptor, Nature, 352 (1991) 725-728.

23. Knowles, R.G. and Moncada, S., Nitric oxide synthases in mammals, Biochem.J., 298 (1994) 249-258.

24. Laube, G. and Veh, R.W., Astrocytes, not neurons, show most prominent staining for spermidine/spermine-like immunoreactivity in adult rat brain, Glia, 19 (1997) 171-179.

25. MacLeod, C.L., Finley, K.D. and Kakuda, D.K., $\mathrm{y}^{(+)}$-type cationic amino acid transport: expression and regulation of the mCAT genes, J.Exp.Biol., 196 (1994) 109-121.

26. Malandro, M.S. and Kilberg, M.S., Molecular biology of mammalian amino acid transporters, Ann.Rev.Biochem., 65 (1996) 305-336.

27. Nakamura, H., Saheki, T., Ichiki, H., Nakata, K. and Nakagawa, S., Immunocytochemical localization of argininosuccinate synthetase in the rat brain, J.Comp.Neurol., 312 (1991) 652679.

28. Nakamura, H., Saheki, T. and Nakagawa, S., Differential cellular localization of enzymes of L-arginine metabolism in the rat brain, Brain Res., 530 (1990) 108-112. 
29. Nakamura, H., Yada, T., Saheki, T., Noda, T. and Nakagawa, S., L-argininosuccinate modulates L-glutamate response in acutely isolated cerebellar neurons of immature rat, Brain Res., 539 (1991) 312-315.

30. Palmer, R.M., Ashton, D.S. and Moncada, S., Vascular endothelial cells synthesize nitric oxide from L-arginine, Nature, 333 (1988) 664-666.

31. Paxinos, G. and Watson, C. (Eds), The rat brain in stereotaxic coordinates, $2^{\text {nd }}$ edition, Academic Press Limited, London, 1986, 260 pp.

32. Rao, V.L., Audet, R.M. and Butterworth, R.F., Increased nitric oxide synthase activities and L- $\left[{ }^{3} \mathrm{H}\right]$ arginine uptake in brain following portacaval anastomosis, J.Neurochem., 65 (1995) 677-678.

33. Ratnakumari, L., Qureshi, I.A., Butterworth, R.F., Marescau, B. and De, D.P., Argininerelated guanidino compounds and nitric oxide synthase in the brain of ornithine transcarbamylase deficient spf mutant mouse: effect of metabolic arginine deficiency, Neurosci.Lett., 215 (1996) 153-156.

34. Ratner, S., Morell, H. and Carvalho, A.P., Enzymes of arginine metabolism in brain, Arch.Biochem.Biophys., 91 (1960) 280-289.

35. Schmidlin, A. and Wiesinger, H., Transport of L-arginine in cultured glial cells, Glia, 11 (1994) 262-268.

36. Schmidlin, A. and Wiesinger, H., Argininosuccinate synthetase - Localization in astrocytes and role in the production of glial nitric oxide, Glia, 24 (1998) 428-436.

37. Son, H., Hawkins, R.D., Martin, K., Kiebler, M., Huang, P.L., Fishman, M.C., and Kandel, E.R., Long-term potentiation is reduced in mice that are doubly mutant in endothelial and neuronal nitric oxide synthase, Cell, 87 (1996) 1015-1023.

38. Stanarius, A., Topel, I., Schulz, S., Noack, H. and Wolf, G., Immunocytochemistry of endothelial nitric oxide synthase in the rat brain: a light and electron microscopical study using the tyramide signal amplification technique, Acta Histochemica, 99 (1997) 411-429.

39. Stevens, B.R., Kakuda, D.K., Yu, K., Waters, M., Vo, C.B. and Raizada, M.K., Induced nitric oxide synthesis is dependent on induced alternatively spliced CAT-2 encoding Larginine transport in brain astrocytes, J.Biol.Chem., 271 (1996) 24017-24022.

40. Stoll, J., Wadhwani, K.C. and Smith, Q.R., Identification of the cationic amino acid transporter (System $\mathrm{y}^{+}$) of the rat blood-brain barrier, J.Neurochem., 60 (1993) 1956-1959.

41. Surh, L.C., Morris, S.M., O'Brien, W.E. and Beaudet, A.L., Nucleotide sequence of the cDNA encoding the rat argininosuccinate synthetase, Nuc.Acids Res., 16 (1988) 9352.

42. Takiguchi, M. and Mori, M., Transcriptional regulation of genes for ornithine cycle enzymes, Biochem.J., 312 (1995) 649-659.

43. Vernet, D., Bonavera, J.J., Swerdloff, R.S., Gonzalez-Cadavid, N.F. and Wang, C., Spontaneous expression of inducible nitric oxide synthase in the hypothalamus and other brain regions of aging rats, Endocrinology, 139 (1998) 3254-3261. 
44. Vockley, J.G., Jenkinson, C.P., Shukla, H., Kern, R.M., Grody, W.W. and Cederbaum, S.D., Cloning and characterization of the human type II arginase gene, Genomics, 38 (1996) 118-123.

45. Weldon, D.T., Maggio, J.E. and Mantyh, P.W., New insights into the neuropathology and cell biology of Alzheimer's disease, Geriatrics, 52 Suppl 2 (1997) S13-S16.

46. Westergaard, N., Beart, P.M. and Schousboe, A., Transport of L- $\left[{ }^{3} \mathrm{H}\right]$ arginine in cultured neurons: characteristics and inhibition by nitric oxide synthase inhibitors, J.Neurochem., 61 (1993) 364-367. 


\section{Figure legends:}

Figure 1: Citrulline-NO cycle, arginine uptake and metabolism. A I+II: arginase I and II, AL: argininosuccinate lyase, Arg: arginine, AS: argininosuccinate synthetase, ASA: argininosuccinic acid, Asp: aspartate, CAT: cationic amino acid transporter, Cit: citrulline, Fum: fumarate, NO: nitric oxide, NOS: nitric oxide synthase, Orn: ornithine.

Figure 2: Expression of CAT1, CAT3, AS, AL, nNOS and AII in the telencephalic cortex. a-c: Low magnification of a sagittal section of rat telencephalic cortex. d-o: enlargement of layers II/III of cortex, as localized by rectangle pictured in 2-b. Neuronal immunostaining for MAP2 (a), ISH antisense staining for CAT1 (b,d), CAT3 (f), AS (c,h), AL (j), nNOS (l) and AII (n), ISH sense staining for CAT1 (e), CAT3 (g), AS (i), AL (k), nNOS (m) and AII (o). In2-c, the deepest cells of layer VI are indicated by an arrowhead. In panels 2-d to 2-n, neurons are indicated by arrowheads and astrocytes by arrows. 2-h and 2-1 are adjacent sections stained for AS (h) and nNOS (l), showing two neurons with colocalized AS and nNOS mRNAs. Bar: $100 \mu \mathrm{m}$.

Figure 3: Expression of CAT1, CAT3, AS, AL, nNOS and AII in the hippocampus and basal ganglia. a-b: Low magnification of a sagittal section of rat hippocampus. c-j: Enlargement of dentate gyrus and hilus, localized by the rectangle outlined in 2-a. k-n: Enlargement of basal ganglia including nucleus accumbens and island of Calleja. ISH antisense staining for CAT1 (a,e,k), CAT3 (f,l), AS (g), AL (h,m), nNOS (i) and AII (b,j,n). Neuronal immunostaining for MAP2 (c) and astrocytic immunostaining for GFAP (d). From 3-c to 3-1, neurons are indicated by arrowheads and astrocytes by arrows. ca1-3: Ammon's horn 1-3, dg: dentate gyrus, ic: island of Calleja, na: nucleus accumbens. Bar: $100 \mu \mathrm{m}$.

Figure 4: Expression of CAT1, CAT3, AS, AL, nNOS and AII in medulla and cerebellum. a,b: High magnification of a sagittal section in rat gigantocellular nucleus of the reticular formation. c-h: high magnification of cerebellar cortex. ISH antisense staining for CAT1 (a,c), CAT3 (d), AS (b,f), AL (e), nNOS (g) and AII (h). From 4-b to 4-h, Purkinje cells are indicated by arrowheads and astrocytes/oligodendrocytes of white matter by arrows. gr: granular layer, mol: molecular layer, wm: white matter. Bar: $100 \mu \mathrm{m}$. 
Table 1

Differential expression of CAT-1, CAT-3, AS, AL, nNOS and A-II in the adult rat brain

- : absent; \pm : barely detectable; + : weak expression; ++ : moderate expression; $++t$ : strong expression; ++++ : very strong expression Levels of transcripts observed by optical microscopy are indicated by - and + signs, which do not represent a strict linear measure of mRNA levels. If only scattered cells express the gene in one area, the expression is given between brackets.

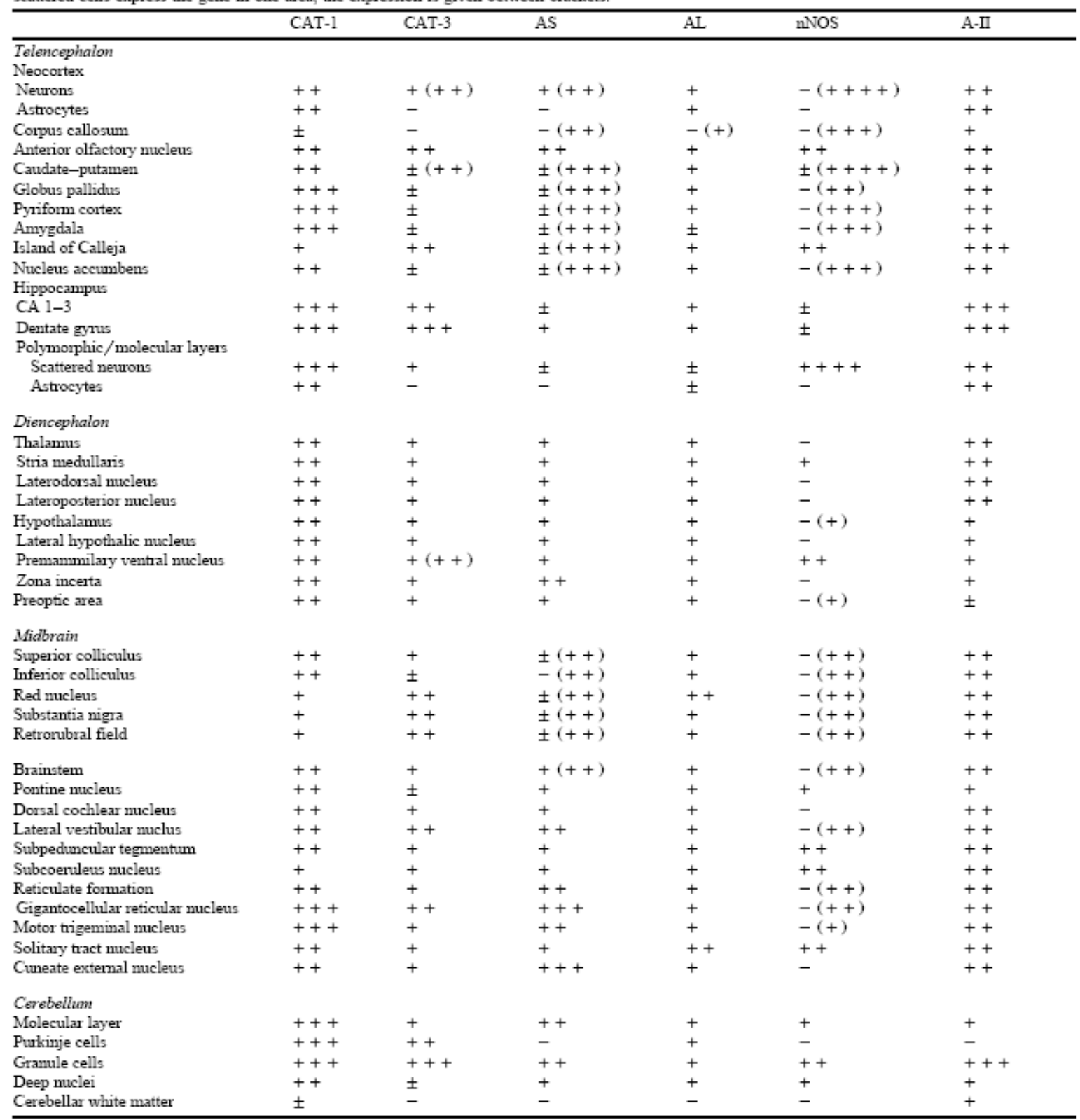


Table 2: Summary of CAT1, CAT3, AS, AL, nNOS and AII expression in the rat brain.

\begin{tabular}{|c|c|c|c|c|c|c|c|}
\hline & & CAT1 & CAT3 & AS & $\mathrm{AL}$ & nNOS & AII \\
\hline \multirow[t]{4}{*}{ Neurons } & telencephalon, diencephalon, brainstem & + & + & + & + & & + \\
\hline & $\begin{array}{l}\text { scattered neurons (telencephalon, midbrain, brainstem), } \\
\text { cerebellar cortex (molecular and granular layers) }\end{array}$ & + & + & + & + & + & + \\
\hline & midbrain, brainstem & + & + & & + & & + \\
\hline & $\begin{array}{l}\text { Purkinje cells } \\
\end{array}$ & + & + & & + & & \\
\hline \multirow[t]{2}{*}{ Astrocytes } & grey matter & + & & & + & & + \\
\hline & white matter & + & & & & & + \\
\hline Oligodendrocytes & & + & & & & & + \\
\hline
\end{tabular}

Main structures for specific patterns of expression are indicated. 
Figure 1 :

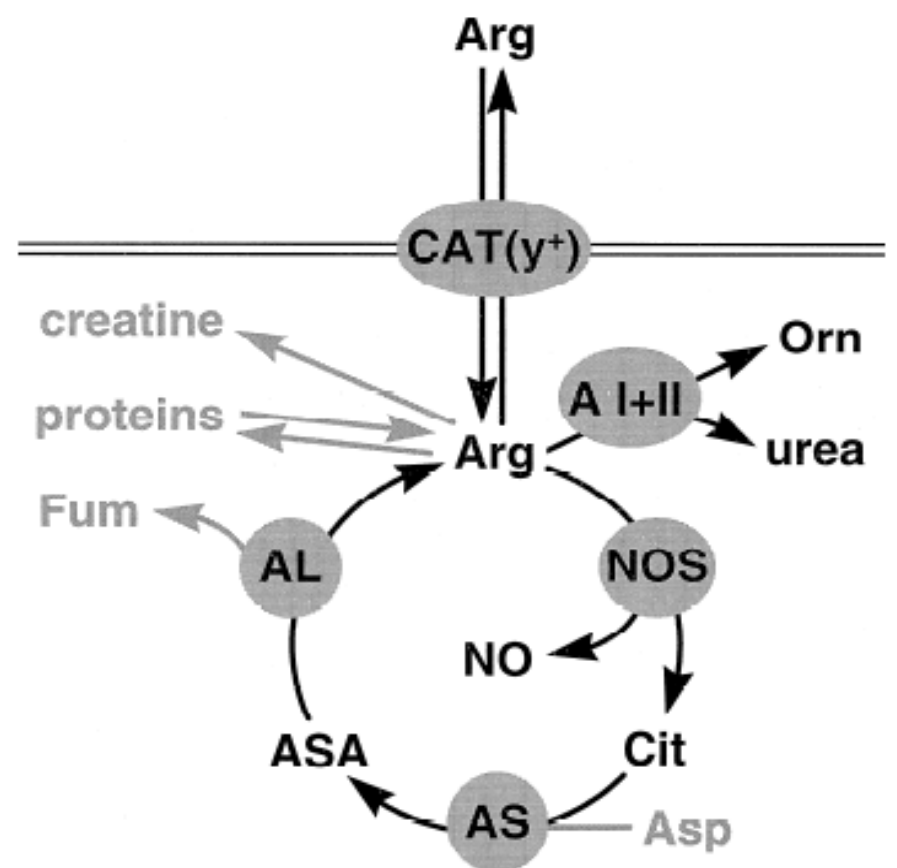


Figure 2 :

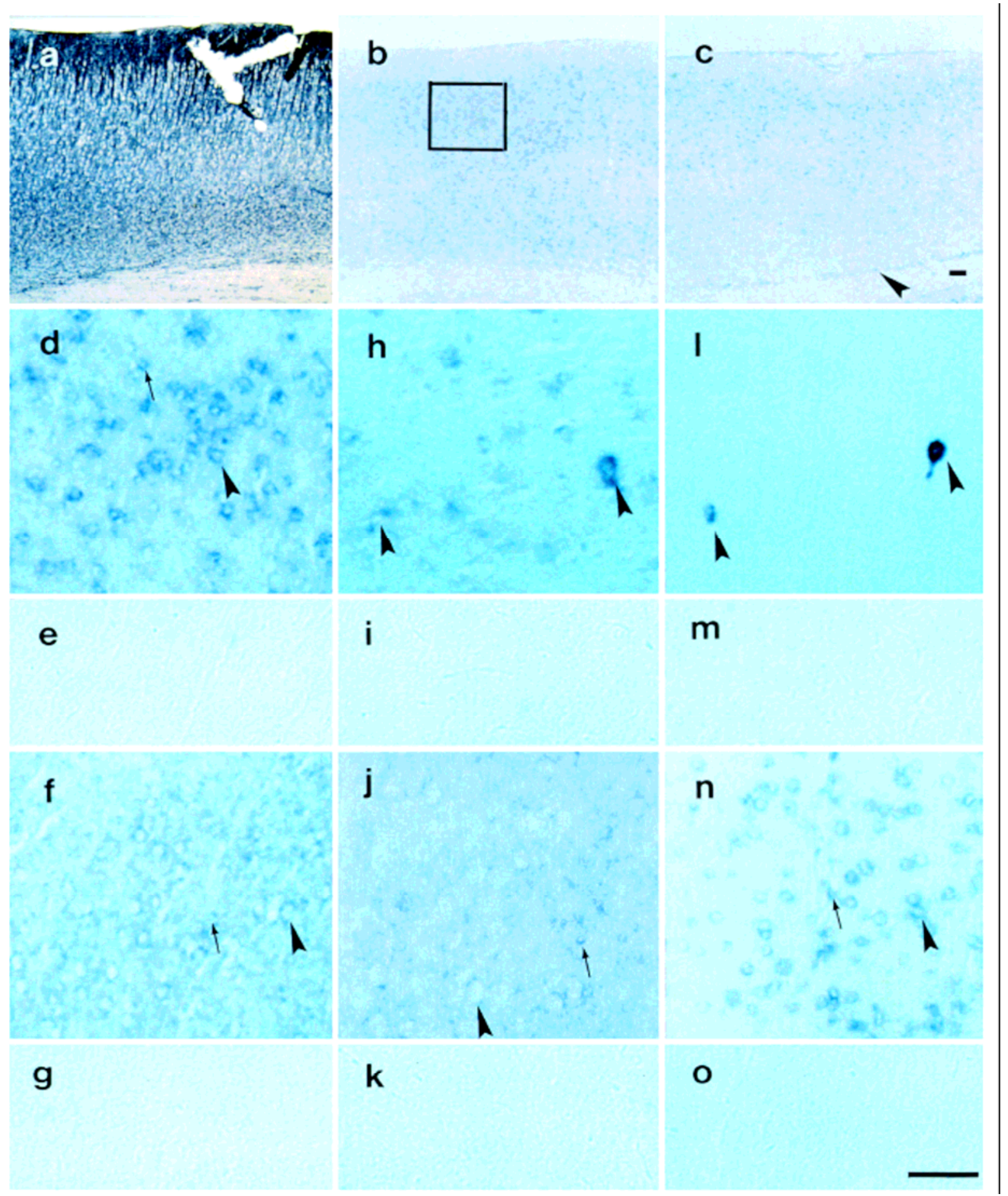


Figure 3 :

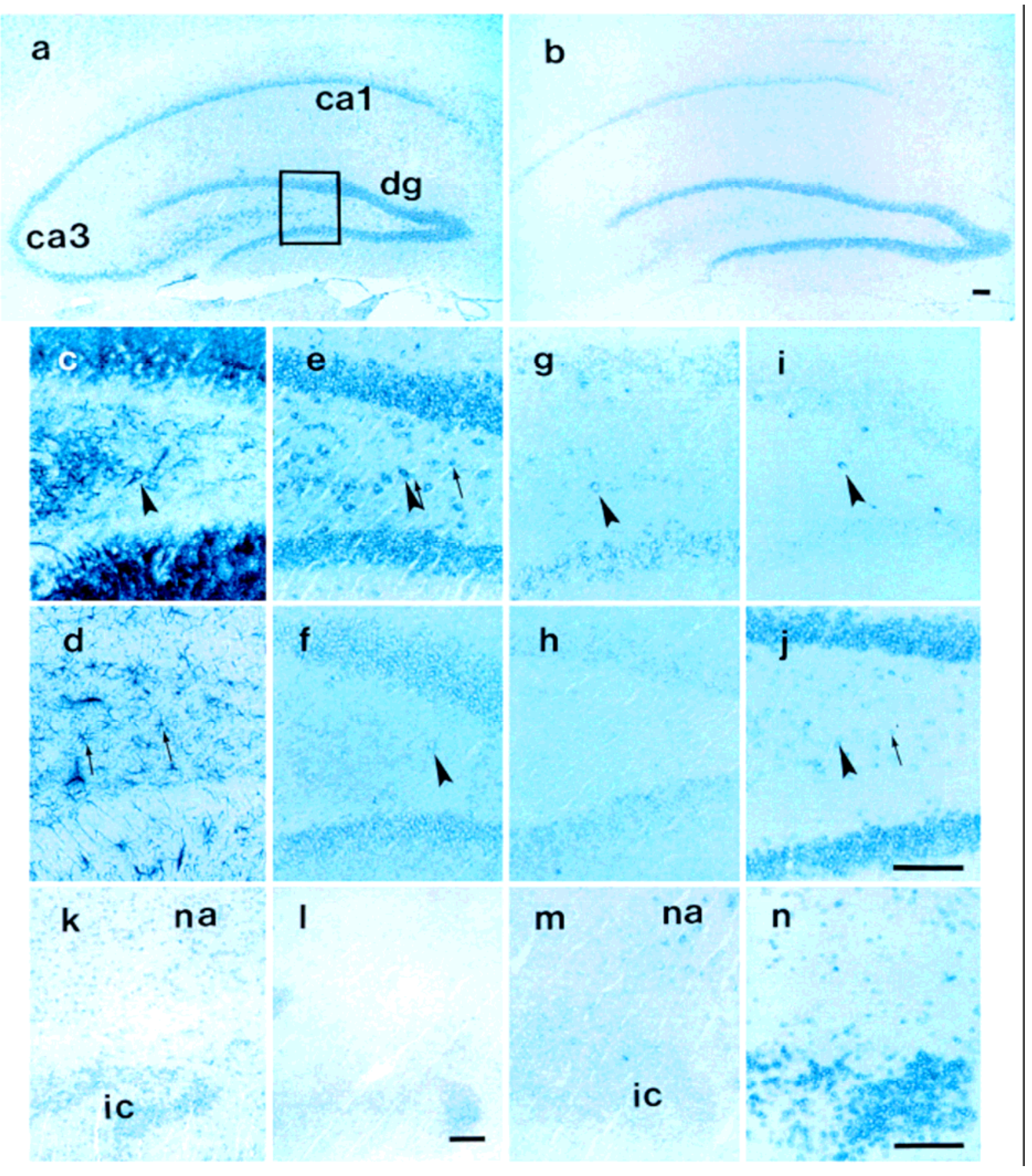


Figure 4 :

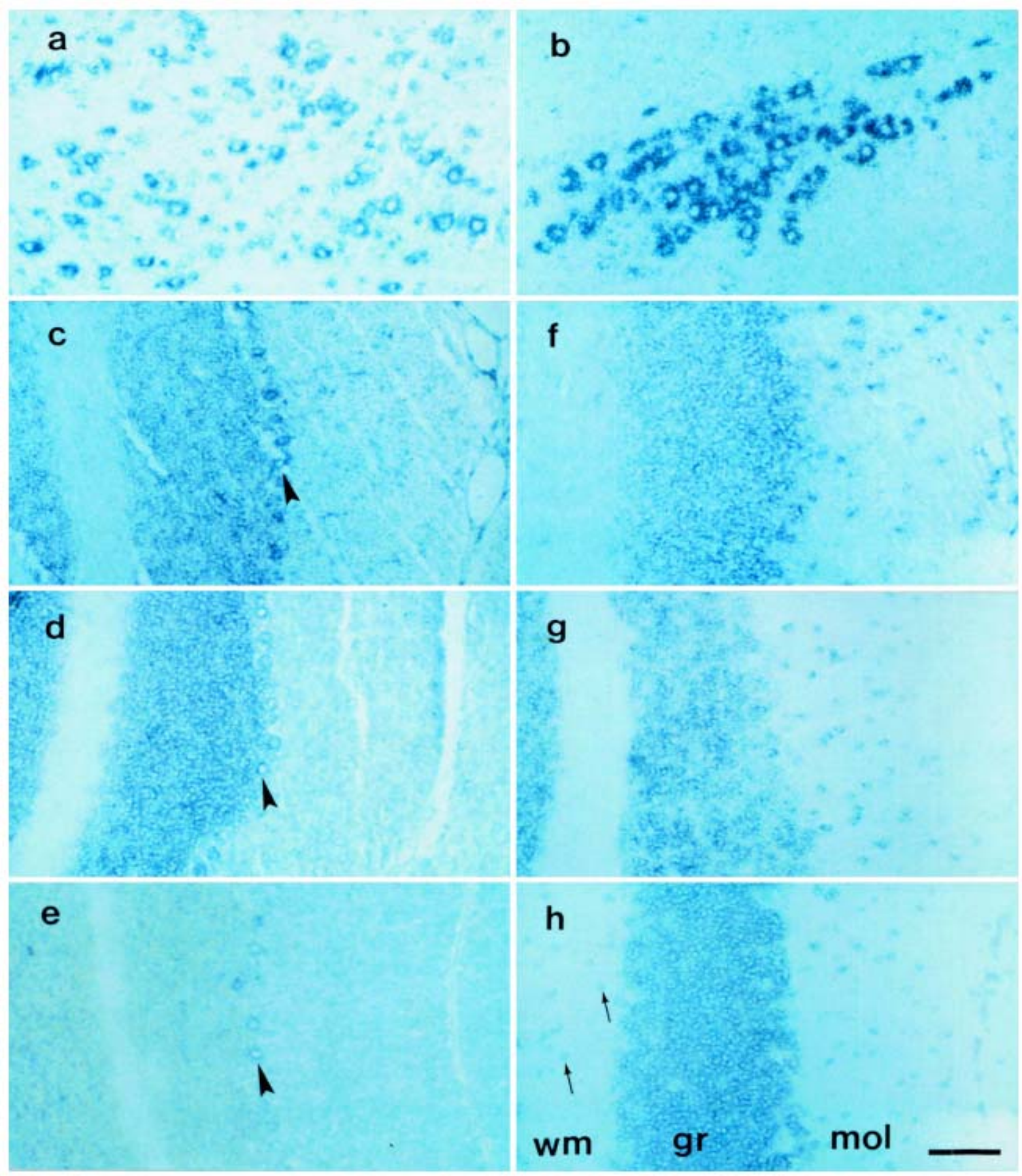

